\title{
Charting molecular free-energy landscapes with an atlas of collective variables
}

Cite as: J. Chem. Phys. 145, 174109 (2016); https://doi.org/10.1063/1.4966262

Submitted: 13 March 2016 • Accepted: 05 October 2016 • Published Online: 03 November 2016

\footnotetext{
(D) Behrooz Hashemian, (D) Daniel Millán and (D) Marino Arroyo
}

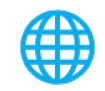

\section{ARTICLES YOU MAY BE INTERESTED IN}

Modeling and enhanced sampling of molecular systems with smooth and nonlinear datadriven collective variables

The Journal of Chemical Physics 139, 214101 (2013); https://doi.org/10.1063/1.4830403

Time-lagged autoencoders: Deep learning of slow collective variables for molecular kinetics The Journal of Chemical Physics 148, 241703 (2018); https://doi.org/10.1063/1.5011399

Enhanced sampling in molecular dynamics

The Journal of Chemical Physics 151, 070902 (2019); https://doi.org/10.1063/1.5109531

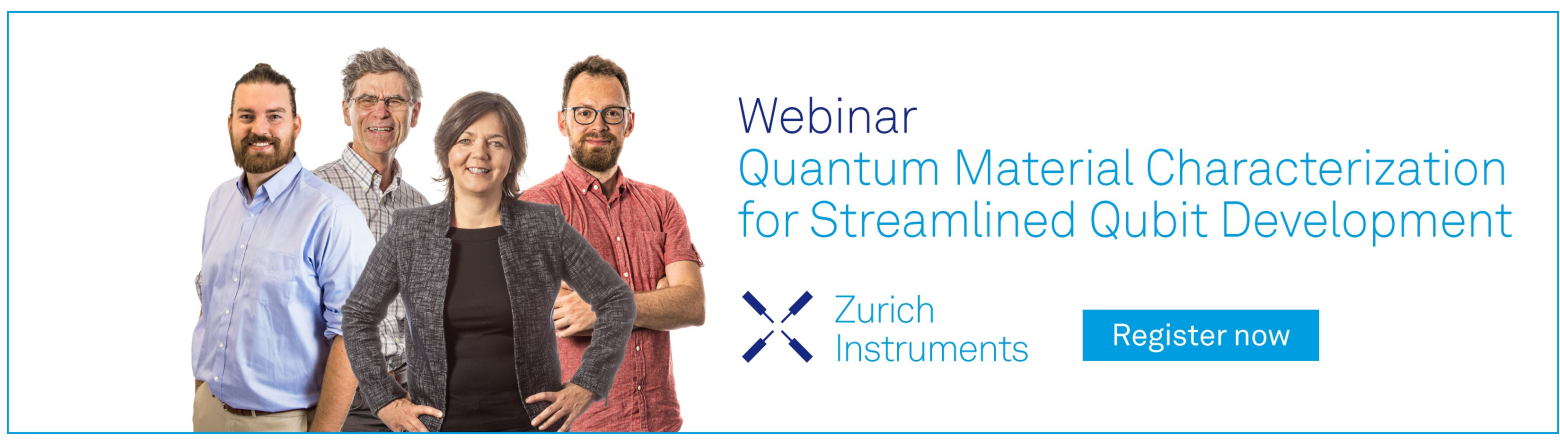




\title{
Charting molecular free-energy landscapes with an atlas of collective variables
}

\author{
Behrooz Hashemian, Daniel Millán, and Marino Arroyoa) \\ LaCàN, Universitat Politècnica de Catalunya-BarcelonaTech, Barcelona, Spain
}

(Received 13 March 2016; accepted 5 October 2016; published online 3 November 2016)

\begin{abstract}
Collective variables (CVs) are a fundamental tool to understand molecular flexibility, to compute free energy landscapes, and to enhance sampling in molecular dynamics simulations. However, identifying suitable CVs is challenging, and is increasingly addressed with systematic data-driven manifold learning techniques. Here, we provide a flexible framework to model molecular systems in terms of a collection of locally valid and partially overlapping CVs: an atlas of CVs. The specific motivation for such a framework is to enhance the applicability and robustness of CVs based on manifold learning methods, which fail in the presence of periodicities in the underlying conformational manifold. More generally, using an atlas of CVs rather than a single chart may help us better describe different regions of conformational space. We develop the statistical mechanics foundation for our multi-chart description and propose an algorithmic implementation. The resulting atlas of data-based CVs are then used to enhance sampling and compute free energy surfaces in two model systems, alanine dipeptide and $\beta$-D-glucopyranose, whose conformational manifolds have toroidal and spherical topologies. Published by AIP Publishing. [http://dx.doi.org/10.1063/1.4966262]
\end{abstract}

\section{INTRODUCTION}

Collective variables (CVs) provide a coarse-grained, lowdimensional description of molecular conformations, and thus help us rationalize many molecular systems including atom clusters, ${ }^{1-3}$ small molecules, ${ }^{4-6}$ polypeptides, ${ }^{7}$ or proteins. ${ }^{8-10}$ If CVs are able to separate the metastable states of the system, then they can be used to enhance sampling in molecular simulations and to efficiently compute free energy surfaces (FES). ${ }^{11}$ Furthermore, since CVs often represent the slowly relaxing degrees of freedom of the system, they may be the basis of reduced models for conformational dynamics. ${ }^{12,13} \mathrm{~A}$ poor selection of the CVs, however, mixes metastable states and results in hysteresis and lack of convergence of enhanced sampling algorithms. ${ }^{14,15}$

Because the identification of suitable $\mathrm{CV}$ s for a given system is a major challenge, there has been an effort over the last decade to develop strategies based on machine learning, more specifically dimensionality reduction methods, ${ }^{16}$ to find systematically such low-dimensional representations. The most widespread dimensionality reduction method is principal component analysis (PCA), a linear method that selects optimal projection directions to maximize variance. PCA is the basis of the essential dynamics approach to analyze collective motions of biomolecules, ${ }^{8,17-20}$ and can be used to enhance sampling in molecular dynamics. ${ }^{21,22}$

Linear methods, such as PCA, however, fail to identify nonlinear correlations in datasets of molecular conformation, e.g., as a result of bond rotations or steric interactions..$^{23-25}$ To better capture the nonlinear low-dimensional geometry of the accessible region in conformational space, the more

\footnotetext{
a) Author to whom correspondence should be addressed. Electronic mail: marino.arroyo@upc.edu
}

recently introduced techniques for nonlinear dimensionality reduction (NLDR) ${ }^{26-28}$ were rapidly applied to understand molecular trajectories, with the underlying hypothesis that these evolve close to a nonlinear manifold often called intrinsic manifold. ${ }^{9,29-31}$ The idea behind an important subset of NLDR methods is to find a low-dimensional representation or embedding of the set of conformations such that high-dimensional distances are preserved as much as possible. The low-dimensional coordinates of the embedding then become the CVs, provided that a differentiable map is available to represent in low dimensions general conformations (out-of-sample conformations), which are not necessarily those used in the process of identifying the low-dimensional coordinates. ${ }^{32,33}$ The high-dimensional distances can be Euclidean in classic multidimensional scaling, ${ }^{16}$ geodesic distances along the intrinsic manifold,,${ }^{9,26}$ diffusion distances, ${ }^{34,35}$ or distances transformed by a sigmoid function to emphasize intermediate length-scales. ${ }^{7}$ Beyond the nonlinear manifold model, a NLDR method called sketch map was put forth that views the accessible region of configuration space as a network of basins connected by a spiderweb of transition pathways. ${ }^{1,7}$ This view is compatible with the intrinsic manifold model because in principle this network of basins and transition pathways can be embedded in a nonlinear manifold, albeit leaving high-energy regions rarely visited. ${ }^{33}$

Besides using these methods to analyze well-sampled ensembles, a number of methods have been developed to enhance sampling along nonlinear CVs identified by machine learning. ${ }^{6,32,33,36}$ In these approaches, the manifold learning algorithm needs to start with a set of conformations representative of molecular flexibility to identify the underlying CVs, a training set in the language of statistical learning, which in itself may be a significant challenge. This problem, however, is less severe than 
that of obtaining thermodynamically meaningful ensembles. Specialized methods of conformational exploration ${ }^{2}$ have been proposed. Furthermore, we showed in previous work that this initial dataset of conformations can be obtained from a simplified model, easier to sample, or can be refined with enhanced simulation trajectories based on a coarse training set. ${ }^{33}$

Despite promising results, these dimensionality reduction approaches face a fundamental obstacle when dealing with conformational landscapes of non-trivial topology, which are very common, e.g., as a result of cyclic motions around a dihedral angle. Periodicities prevent NLDR algorithms from untangling and displaying coherently in low dimensions the relevant conformational space. Instead, dimensionality reduction methods applied to such systems can mix conformations belonging to distant basins, collapse transition pathways, and severely distort parts of the landscape. This can ruin the value of the resulting CVs. This issue was identified and discussed in Ref. 36, and has plagued a number of applications of NLDR methods to molecular simulation. ${ }^{6,31,35,37,38}$ In Ref. 39, we further examined this issue and showed that it manifests itself irrespective of the NLDR algorithm; we considered a non-metric NLDR method (locally linear embedding ${ }^{27}$ ) and two distance-preserving methods that use different notions of distance (diffusion map $^{34}$ and Isomap ${ }^{26}$ ). While acknowledging the issue, the authors in Ref. 36 did not provide a specific remedy to these topological obstructions in NLDR, but rather proposed an enhanced sampling algorithm not based on the classical notion of $\mathrm{CV}$ to alleviate the consequences of a deficient mapping to low-dimensions.

Here, our goal is to develop a general method capable of dealing with systems of arbitrary topology to realize the potential of manifold learning methods for systematic identification of CVs. The idea behind our method comes from the realization that analogous issues are confronted in cartography. The globe is a two-dimensional manifold without boundary. Furthermore, as a result of Gauss' Egregium theorem, it is not possible to map even a portion of the surface of a sphere onto a plane without distorting it. These are fundamental topological and geometric hurdles to cartography, which are dealt with by mapping the globe using a collection (atlas) of charts, each describing with appropriate detail a region of limited extent. Each chart has boundary and is planar, just like the output of a NLDR algorithm. By reducing the lateral extent of the region covered by a chart, the metric distortion can be reduced arbitrarily. Here, we examine whether this idea to describe geographical landscapes can be transposed to conformational landscapes. Beyond facilitating the application of manifold learning methods to construct CVs, this approach could have broader applicability. After all, it is natural to expect that a complex molecule may be best described by different CVs (different charts) in different regions of its conformational space, where different steric constraints may determine molecular flexibility. With a framework to model molecular systems with an atlas of CVs, each individual chart could be suitably identified with manifold learning methods, and still describe a coherent conformational landscape. Furthermore, the partitioning of configuration space into different regions naturally introduce a length-scale, since dimensionality reduction methods can only provide correlations within the extent of the corresponding partition. It has been argued that it is important to observe an intermediate length-scale in the NLDR of complex molecular systems. ${ }^{7}$

However, to our knowledge free energy formalisms and enhanced sampling methods have only been developed considering a single set of CVs. We devote Section II to revisiting the classical free energy formalisms for a system described by an atlas of multiple partially overlapping CVs. This theory shows how to compute seamlessly thermodynamical observables over a multiple-chart description, and also imposes constraints on the connection between adjacent CVs for a meaningful statistical mechanics description. In Section III, we describe a specific computational method to implement the atlas of CVs, although the formalism can be applied to other data-driven approaches to build CVs and to other enhanced sampling strategies. Our method is based on the intrinsic manifold model. We identify the nonlinear manifold around which conformations cluster with the Isomap NLDR algorithm. ${ }^{26}$ The multiple chart description is then built using systematic graph-partitioning algorithms combined with the SandCV methodology presented earlier, ${ }^{33}$ which provides a differentiable mapping for out-of-sample conformations onto the reduced space of CVs. In Section III, it is described how to extend naturally an enhanced sampling algorithm from a single set of CVs to an atlas of CVs, focusing on the adaptive biasing force $(\mathrm{ABF})$ method. $^{40}$ As a first step to test the applicability of the atlas of CVs framework to molecular modeling, in Section IV we obtain atlases and perform enhanced sampling simulations to compute the free energy of two model systems: alanine dipeptide and $\beta$-D-glucopyranose. The first of these molecules is a standard benchmark in the field of identification of CVs by statistical/manifold learning. Good CVs in terms of dihedral angles are known for these systems, which provide a basis for comparison with data-driven CVs. Furthermore, the intrinsic manifold on which the conformations evolve has non-trivial topology in both cases (that of a torus and of a sphere), which if improperly dealt with, has led in the past to failures in manifold learning algorithms, see Ref. 39 and references therein.

\section{THEORY}

Before introducing an atlas description of a molecular system, we review the standard formalism based on a single $\mathrm{CV}$. Consider an $\mathrm{N}$-atom system with separable Hamiltonian and potential energy $\mathcal{V}(\boldsymbol{r})$. Mathematically, a $\mathrm{CV}$ is a differentiable mapping taking a conformation $\boldsymbol{r}$ in highdimensional configurational space $D=\mathbb{R}^{3 N}$ and mapping it to $\boldsymbol{\xi}$, a point in low-dimensional space $\mathbb{R}^{d}$, where $d \ll 3 N$. We denote this mapping by $\boldsymbol{\xi}=C_{1}(\boldsymbol{r})$. The free energy along this $\mathrm{CV}$, up to an additive constant, can be defined as

$$
A_{1}(\boldsymbol{\xi})=-\frac{1}{\beta} \ln \int_{D_{1}} e^{-\beta \mathcal{V}(\boldsymbol{r})} \delta\left(C_{1}(\boldsymbol{r})-\boldsymbol{\xi}\right) d \boldsymbol{r},
$$

where $\delta(\cdot)$ is the Dirac delta distribution, ${ }^{11,41}$ and $1 / \beta=k T$ is the Boltzmann constant times temperature. For any given 
value of the collective variable $\boldsymbol{\xi}$, the level set $\mathcal{L}_{1}(\xi)$ is the set of all molecular conformations $\boldsymbol{r}$ that are mapped onto $\xi$, i.e., $C_{1}(\boldsymbol{r})=\boldsymbol{\xi}$. Applying the co-area formula, ${ }^{42}$ the free energy can be written as an integral over the level set $\mathcal{L}_{1}(\xi)$ as

$$
A_{1}(\xi)=-\frac{1}{\beta} \ln \int_{\mathcal{L}_{1}(\xi)} e^{-\beta \mathcal{V}} \operatorname{vol}\left(\mathbf{D} C_{1}\right)^{-1} d \sigma_{\xi},
$$

where $\mathbf{D} C_{1}$ is the Jacobian matrix of the $\mathrm{CV}, d \sigma_{\xi}$ is the volume element of $\mathcal{L}_{1}(\boldsymbol{\xi})$, and $\operatorname{vol}\left(\mathbf{D} C_{1}\right)=\sqrt{\left|\mathbf{D} C_{1} \mathbf{D} C_{1}^{T}\right|}$. $|\cdot|$ stands for the determinant. If the $\mathrm{CV}$ has been chosen appropriately, then the probability distribution along the level sets $\mathcal{L}_{1}(\boldsymbol{\xi})$ should be not too different from a Gaussian, i.e., the system should not exhibit transversal metastability.

Figure 1 illustrates some of these concepts, with the high-dimensional configurational space represented by a box, and the level set of the scalar $\mathrm{CV}, \xi=C_{1}(\boldsymbol{r})$, represented in red. In black, we represent the intrinsic manifold for this system, which passes close to the energy basin within this red level set. It is clear from the figure that, if the transverse energy landscape was complex with multiple basins, exhibiting transverse metastability, then the notion of intrinsic manifold would become less apparent. The intrinsic manifold is not strictly required in the free energy formalism, but will become useful later. In this simple illustration, configuration space in three-dimensional, there is a single collective variable $(d=1)$, hence the intrinsic manifold is one-dimensional, and the level sets are two-dimensional $(3 N-d=2)$. For the molecular systems examined later, the intrinsic manifold is two-dimensional $(d=2)$, whereas the level sets and configuration space are higher-dimensional and cannot be easily visualized.

We are now going to use the collective variable $C_{1}$ to describe the system only in a sub-region $D_{1} \subset D$ of the full configurational space, and use a different $\mathrm{CV}, \boldsymbol{\eta}=C_{2}(\boldsymbol{r})$, in another region of configuration space $D_{2} \subset D$, which partially overlaps with $D_{1}\left(D_{1} \cap D_{2} \neq \emptyset\right)$. Examining Eq. (2), it is clear that the free energies along these two CVs can only be related in the overlapping region if their respective level sets can be mapped to each other. For this to be the case, there must exist a one-to-one transition mapping $\varphi: D_{1} \subset \mathbb{R}^{d} \rightarrow D_{2} \subset \mathbb{R}^{d}$ such

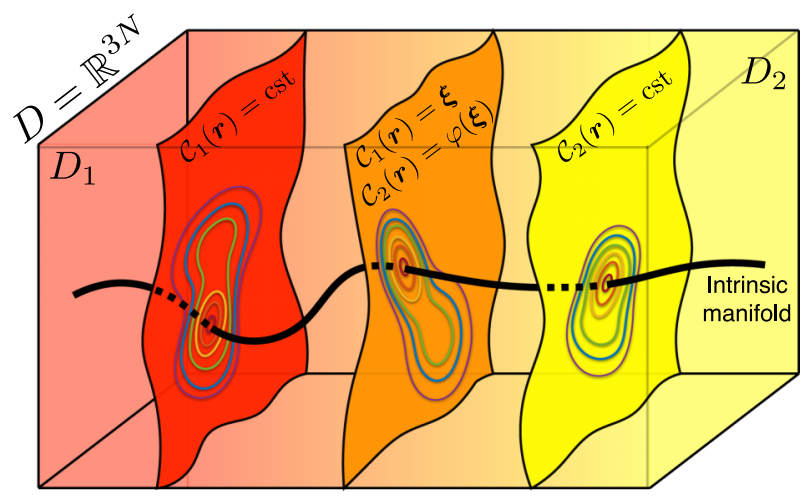

FIG. 1. Graphical illustration of high-dimensional configuration space $D$, described in the left by collective variable $C_{1}$, in the right by $C_{2}$, and in the central overlapping region by both. The surfaces illustrate the level sets. The level set in the middle can be described either as $C_{1}(\boldsymbol{r})=\boldsymbol{\xi}$ or as $C_{2}(\boldsymbol{r})=\varphi(\boldsymbol{\xi})$. that

$$
C_{2}(\boldsymbol{r})=\varphi \circ C_{1}(\boldsymbol{r})
$$

for $\boldsymbol{r} \in D_{1} \cap D_{2}$. If this is the case, then the hyper-surface given by $\mathcal{C}_{1}(\boldsymbol{r})=\boldsymbol{\xi}$ can also be characterized using the second $\mathrm{CV}$ as $C_{2}(\boldsymbol{r})=\varphi(\xi)$.

We assume for now that such a transition mapping $\boldsymbol{\eta}=\varphi(\xi)$ exists and leave for later an explicit construction. This mapping provides a connection between different CVs and a means to build a meaningful global statistical mechanics description of the system. Indeed, we can write Eq. (2) for the second $\mathrm{CV}$ as

$$
A_{2}(\boldsymbol{\eta})=-\frac{1}{\beta} \ln \int_{\mathcal{L}_{2}(\boldsymbol{\eta})} e^{-\beta \mathcal{V}} \operatorname{vol}\left(\mathbf{D} C_{2}\right)^{-1} d \boldsymbol{\sigma}_{\boldsymbol{\eta}} .
$$

Using the chain rule on Eq. (3), we have the relation between Jacobian matrices $\mathbf{D} C_{2}(\boldsymbol{\eta})=\mathbf{D} \varphi(\boldsymbol{\xi}) \mathbf{D} \mathcal{C}_{1}(\boldsymbol{\xi})$. Furthermore, we note that $\operatorname{vol}\left(\mathbf{D} \varphi \mathbf{D} C_{1}\right)=|\mathbf{D} \varphi| \operatorname{vol}\left(\mathbf{D} C_{1}\right){ }^{43}$ Thus, recalling that $\mathcal{L}_{2}(\boldsymbol{\eta})=\mathcal{L}_{1}(\boldsymbol{\xi})$, we can perform a change of variables in Eq. (4) to obtain

$$
\begin{aligned}
A_{2}(\boldsymbol{\eta}) & =-\frac{1}{\beta} \ln \int_{\mathcal{L}_{1}(\xi)} e^{-\beta \mathcal{V}}\left[|\mathbf{D} \varphi| \operatorname{vol}\left(\mathbf{D} C_{1}\right)\right]^{-1} d \sigma_{\xi} \\
& =-\frac{1}{\beta} \ln \int_{\mathcal{L}_{1}(\xi)} e^{-\beta \mathcal{V}} \operatorname{vol}\left(\mathbf{D} C_{1}\right)^{-1} d \sigma_{\xi}+\frac{1}{\beta} \ln |\mathbf{D} \varphi(\xi)| \\
& =A_{1}(\xi)+\frac{1}{\beta} \ln |\mathbf{D} \varphi(\xi)|,
\end{aligned}
$$

where we have used the fact that $\mathbf{D} \varphi$ only depends on $\boldsymbol{\xi}$ and hence can be factored outside of the integral.

Equation (5) relates the free energies along the two CVs in the overlapping region. This expression highlights the fact that the free energy is subjective in that it depends on the specific parametrization along a collective variable, although observables computed from it are objective. ${ }^{41,44}$ The last term in Eq. (5) is reminiscent of a Fixman potential. ${ }^{42,45}$ Recalling that both $A_{1}$ and $A_{2}$ are computed up to an additive constant, Eq. (5) provides a compatibility relation between these constants.

Now, let us compute thermodynamic observables, such as relative probabilities of states, in a multiple CV framework. Consider two conformations characterized by regions $A$ and $B$ belonging to $D_{1}$ and $D_{2}$, respectively. Consider also an auxiliary state $C \subset D_{1} \cap D_{2}$. The relative probabilities between states $A$ and $C$ and between $C$ and $B$ can be computed as

$$
\frac{p(A)}{p(C)}=\frac{\int_{C_{1}(A)} e^{-\beta A_{1}(\xi)} d \xi}{\int_{\mathcal{C}_{1}(C)} e^{-\beta A_{1}(\xi)} d \xi}, \quad \frac{p(C)}{p(B)}=\frac{\int_{C_{2}(C)} e^{-\beta A_{2}(\boldsymbol{\eta})} d \boldsymbol{\eta}}{\int_{C_{2}(B)} e^{-\beta A_{2}(\boldsymbol{\eta})} d \boldsymbol{\eta}} .
$$

Consequently, the relative probability between $A \subset D_{1}$ and $B \subset D_{2}$ takes the form

$$
\frac{p(A)}{p(B)}=\frac{\int_{C_{1}(A)} e^{-\beta A_{1}(\boldsymbol{\xi})} d \boldsymbol{\xi}}{\int_{C_{2}(B)} e^{-\beta A_{2}(\boldsymbol{\eta})} d \boldsymbol{\eta}} \underbrace{\frac{\int_{\mathcal{C}_{2}(C)} e^{-\beta A_{2}(\boldsymbol{\eta})} d \boldsymbol{\eta}}{\int_{\mathcal{1}_{1}(C)} e^{-\beta A_{1}(\boldsymbol{\xi})} d \boldsymbol{\xi}}}_{=1} .
$$

Invoking Eq. (5) and using the change of variable formula, it follows that the second term in this equation is 1 . Consequently $p(A) / p(B)$ can be computed without reference to state $C$. This shows that the statistical mechanics of the system can be seamlessly formulated across multiple CVs. The arguments 
drawn here extend directly to an atlas of multiple partially overlapping CVs.

Next, we examine the impact of having multiple CVs on accelerated free energy calculations. In many enhanced sampling methods, such as metadynamics ${ }^{46}$ or the adaptive biasing force (ABF) method, ${ }^{40}$ an approximation to the thermodynamic force at $\xi=C(\boldsymbol{r})$ along the $\mathrm{CV}$, denoted by $\boldsymbol{f}(\boldsymbol{\xi}) \approx-\mathbf{D} A(\boldsymbol{\xi})$ (or $f^{i} \approx-\partial A / \partial \xi^{i}$ with $i=1, \ldots, d$ ), is mapped onto a force on the atoms that biases the dynamics given by

$$
F^{j}(\boldsymbol{r})=\sum_{i=1}^{d} \frac{\partial C^{i}}{\partial r^{j}}(\boldsymbol{r}) f^{i}(C(\boldsymbol{r})),
$$

with $j=1, \ldots, 3 N$, or in matrix notation

$$
\boldsymbol{F}(\boldsymbol{r})=\mathbf{D} C^{T}(\boldsymbol{r})[\boldsymbol{f} \circ \mathcal{C}(\boldsymbol{r})] .
$$

In the ideal situation, $\boldsymbol{f}$ is close to $-\mathbf{D} A(\xi)$, and the enhanced sampling trajectory undergoes nearly free diffusion along the CVs irrespective of free energy barriers. Consider now a two-CV atlas and $\boldsymbol{r} \in D_{1} \cap D_{2}$. Assuming that $f_{\alpha} \approx-\mathbf{D} A_{\alpha}$ $(\alpha=1,2)$, a direct calculation shows that as a result of Eq. (5) the biasing forces given by each $\mathrm{CV}$ differ by a geometric term

$$
\boldsymbol{F}_{1} \approx \boldsymbol{F}_{2}-\frac{1}{\beta} \mathbf{D} C_{1}^{T}[\mathbf{D}(\ln |\mathbf{D} \varphi|)] .
$$

From this equation it follows that when $\boldsymbol{r}$ is in the overlapping region during an enhanced sampling MD simulation, the algorithm must make a choice for the biasing force $\boldsymbol{F}(\boldsymbol{r})$, which cannot simultaneously represent the thermodynamic force along $C_{1}$ and $C_{2}$. As we illustrate in the numerical examples, however, this fact does not seem of any practical importance because the geometric correction in the equation above is very small.

\section{METHOD}

We have shown that it is possible to build a coherent statistical mechanics description of a molecule in terms of an atlas of CVs, provided that transition mappings exist as in Eq. (3). However, two questions remain: (1) how to construct practically such an atlas of CVs, and (2) how to bias a MD simulation along such an atlas. Here, we present an implementation of the atlas of CVs framework based on a specific method that we presented earlier to build smooth and nonlinear datadriven collective variables (SandCV), ${ }^{33}$ which we combine with enhanced sampling using the adaptive biasing force (ABF) method. ${ }^{40}$ However, the framework is general enough to accommodate many other variants, as we discuss later.

\section{A. Building an atlas of collective variables}

The SandCV method resorts to the intrinsic manifold model for molecular systems. In this model, it is assumed that the dynamics of the molecule take place close to a manifold (in general nonlinear) of dimension $d$ embedded in the $3 N$ - dimensional conformational space. Although it may seem a rather strong assumption, this notion has been shown to be useful in a number of systems. ${ }^{9,29,30,33,47}$ This systematic and robust method is graphically summarized in
Figure 2. We note that a NLDR method provides a way to embed in low-dimensions the configurations within a training set of configurations, but does not provide immediately a smooth mapping of a generic out-of-sample configuration $\boldsymbol{r}$ onto low-dimensional space, that is, a proper CV as needed in most enhanced sampling methods. To construct this mapping, we first parametrize the intrinsic manifold as $r=\mathcal{M}(\boldsymbol{\xi})$ (see Appendix A or Ref. 33 for details), using standard leastsquares fitting and smooth basis functions. Then, starting from a conformation in high-dimensions $\boldsymbol{r}$ (in general off the manifold), we first compute its closest-point projection $\mathcal{P}(\boldsymbol{r})$ onto the intrinsic manifold and then find the pre-image through the mapping $\mathcal{M}$, i.e., $\xi=C(\boldsymbol{r})=\mathcal{M}^{-1} \circ \mathcal{P}(\boldsymbol{r})$. This collective variable is differentiable under mild conditions and in practice, its evaluation requires solving a straightforward nonlinear minimization problem with $d$ unknowns. Furthermore, its derivative $\mathbf{D C}$ can be computed explicitly. ${ }^{33}$

To implement the atlas of CVs, we start by splitting the training set into a few disjoint partitions, as illustrated in Figure 3(a). This partitioning is efficiently performed in high dimensions, by first building a connectivity graph of the training set based on the $K$-nearest neighbors to each conformation, and then using systematic graph partitioning algorithms ${ }^{48}$ combined with a slight enlargement of the partitions to provide overlap, see Appendix A. This partitioning is performed recursively until each patch is flat enough and devoid of topological obstructions to be tractable by nonlinear dimensionality methods. ${ }^{49}$ Then, each of the training sub-sets is embedded separately in low-dimensions with the NLDR method of choice, here Isomap.

This procedure is illustrated in more detail in Figure 4 focusing on alanine dipeptide, a standard benchmark with a good known CV in terms of two dihedral angles, Figure 4(a). The training set is visualized in Figure 4(b) using aligned molecular configurations, and in (c) on the surface of a torus, thanks to the dihedral representation. An in-depth discussion about the topology and geometry of the toroidal intrinsic manifold underlying this molecule has been presented elsewhere. ${ }^{39}$ The training sub-sets resulting from the partitioning algorithm are depicted in (e) as aligned molecular configurations, and in (d) on the torus representation. The figure clearly shows how partitioning allows us to decompose a manifold without boundary and with the topology of the torus into four partitions with boundary and the topology of an open set in $\mathbb{R}^{d}$, ideally suited for NLDR. The low-dimensional embeddings of the four partitions are shown in (f).

Now, the SandCV method can be applied separately to each of the partially overlapping partitions to define an atlas of CVs, $\left\{C_{\alpha}\right\}_{\alpha=1 \ldots L}$. Importantly, the proposed method provides $a b$ initio compatible collective variables in the sense of having common level sets in overlap regions, along with the transition mappings. Indeed, suppose that the intrinsic manifold is described by two partially overlapping parametrizations $\mathcal{M}_{1}$ and $\mathcal{M}_{2}$, each of which generating a collective variable. Because of the closest-point construction of SandCV, the level sets $\mathcal{L}_{1}$ and $\mathcal{L}_{2}$ are hyper-planes perpendicular to the intrinsic manifold in its vicinity, see Figure $3(\mathrm{~b})$. Therefore, $C_{1}$ and $C_{2}$ share their level sets in the overlap region and the transition mapping is simply $\varphi=\mathcal{M}_{2}^{-1} \circ \mathcal{M}_{1}$. 


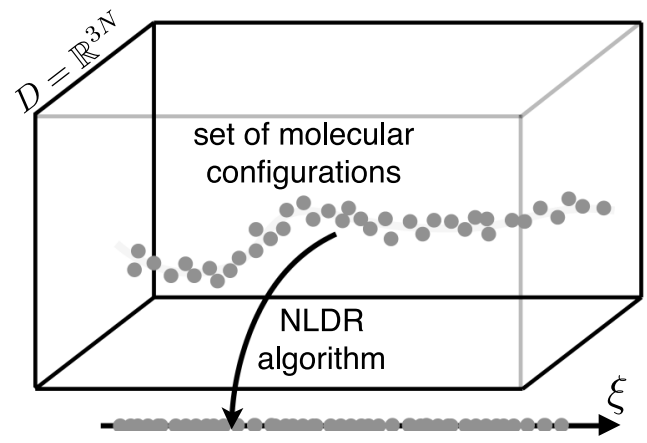

(a) Low-dimensional embedding by dimensionality reduction

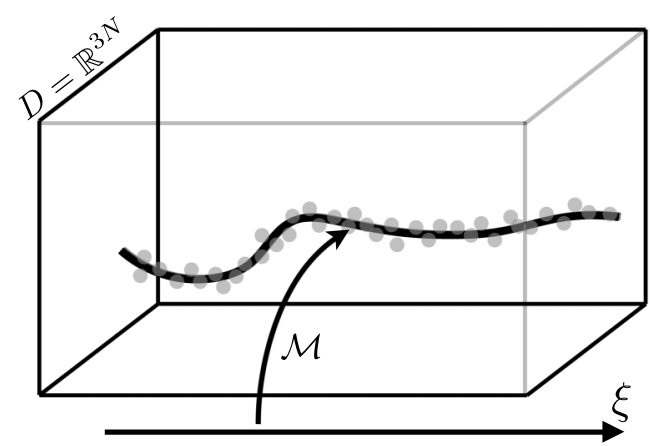

(b) Parametrization of the intrinsic manifold

(c) Projection of an outof-sample configuration r onto CV space

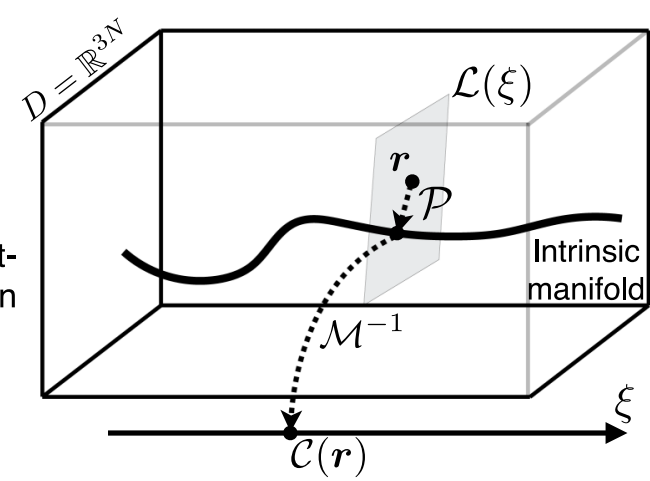

FIG. 2. Graphical summary of the smooth and nonlinear data-driven collective variables (SandCV) method, presented in detail in Ref. 33. (a) Given a training set of molecular configurations (represented as points in high-dimensional space $D$ ), we use a NLDR method (here Isomap) to find a low dimensional embedding of these points. (b) Then, we parametrize the underlying intrinsic manifold with a mapping $\boldsymbol{r}=\mathcal{M}(\xi)$, in an exercise of curve-fitting in higher dimensions. (c) Finally, to define a proper $\mathrm{CV}$, i.e., a differentiable mapping $\boldsymbol{\xi}=\mathcal{C}(\boldsymbol{r})$ from $D$ onto the low-dimensional $\mathrm{CV}$ space, we first map a generic configuration $\boldsymbol{r}$ onto the intrinsic manifold using the closest-point projection $\mathcal{P}$, and then find the low-dimensional pre-image of this point applying $\mathcal{M}^{-1}$. Because of the closest point projection, the level sets $\mathcal{L}(\xi)$ of this $\mathrm{CV}$ are hyperplanes normal to the intrinsic manifold.

Given a generic molecular configuration $r$, one last ingredient is required to locate the active $\mathrm{CV}$ s, i.e., the set of CVs that can be used to represent the configuration in low dimensions. Because of the partial overlap, in general there can be more than one active CV. For this, we resort to a partition of unity subordinate to the geometric partition discussed above and defined in high-dimensional space. See Appendix A for a detailed description of the algorithm, which poses nearly no computational overhead. This partition of unity is simply a collection of non-negative functions $\psi_{\alpha}(\boldsymbol{r})$ that are 1 in the interior of each region $D_{\alpha}$, smoothly decay to zero in the overlap region, and $\sum_{\alpha} \psi_{\alpha}(\boldsymbol{r})=1$. Thus if

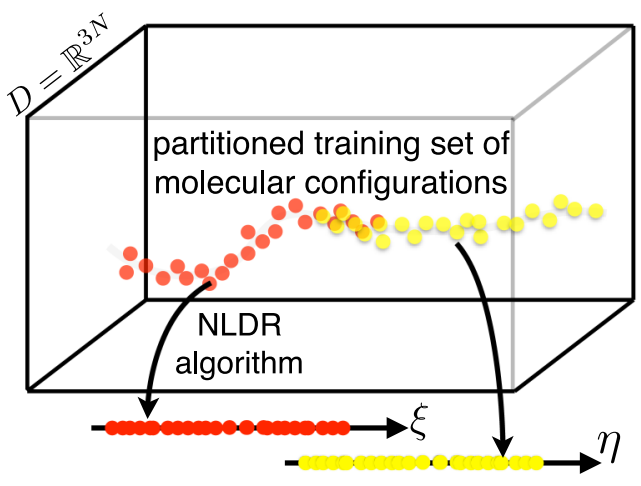

(a) NLDR applied to each partition of the training set

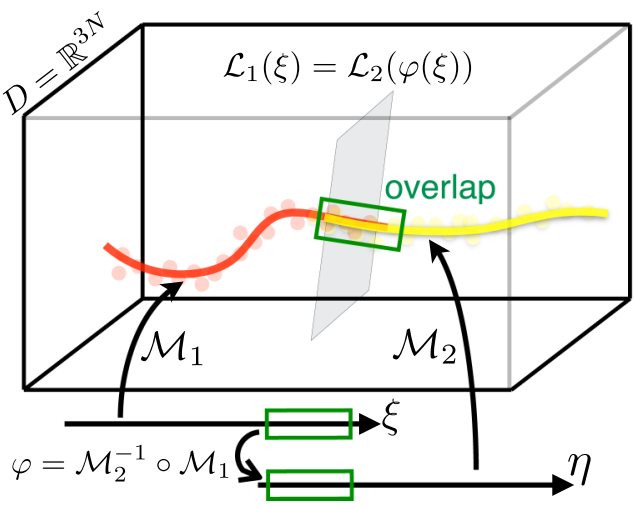

(b) The SandCV method can be applied to each partition and provides explicitly the transition mapping $\varphi$

FIG. 3. Illustration of the proposed method to implement the atlas of CVs. (a) Using systematic graph partitioning methods, the training set is partitioned into simpler sub-sets, here color-coded in red and yellow, and embedded in low-dimensions separately. (b) Then, the SandCV method can be applied separately to each adjacent partition. Importantly, because the CVs in the overlapping region share the level sets, which geometrically are hyperplanes perpendicular to the intrinsic manifold, see Figure 2(c), the transition mapping $\varphi$ can be explicitly defined in terms of the two overlapping parametrizations of the intrinsic manifold. 
(a)

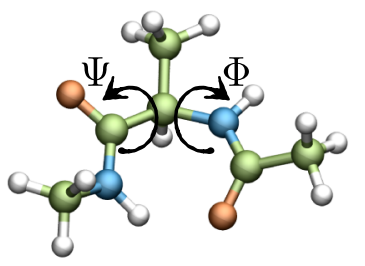

(e) conformations in each
of the four partitions

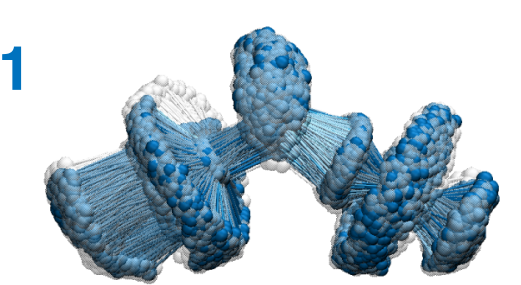

2
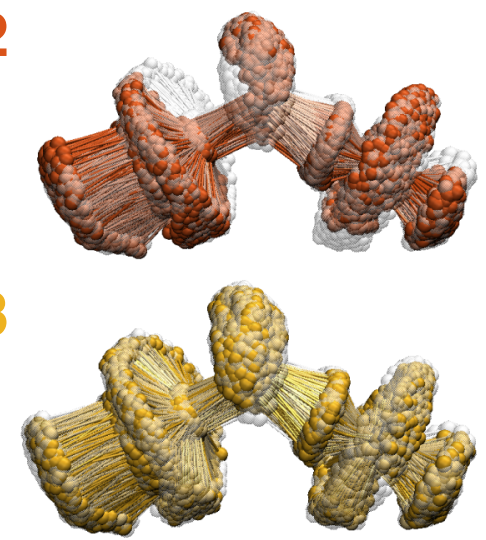

(d)

(b) visualization of the training set
as aligned conformations

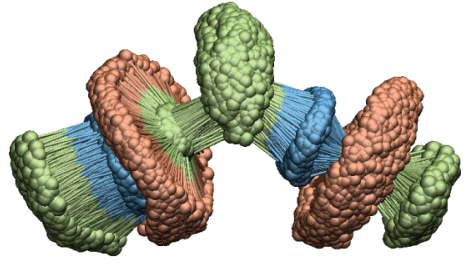

(c) visualization of the training set using dihedral angles on a torus
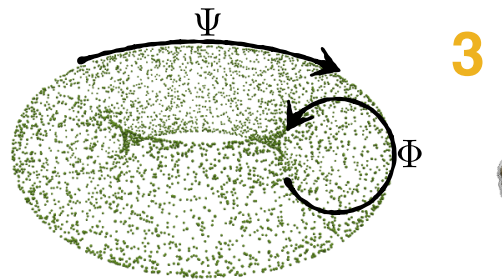

visualization of the four partitions on a torus
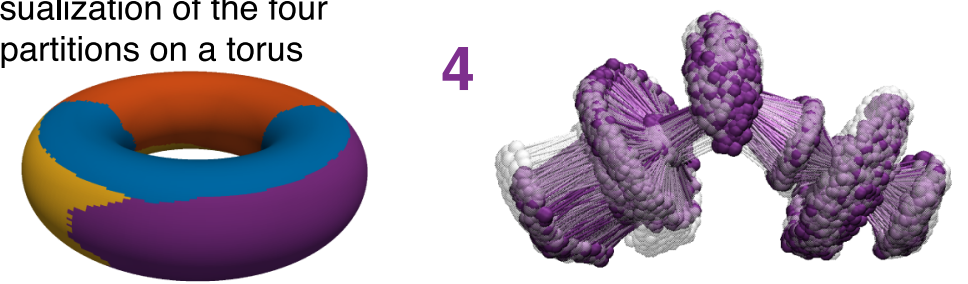

two-dimensional
embeddings obtained

for each partition using NLDR
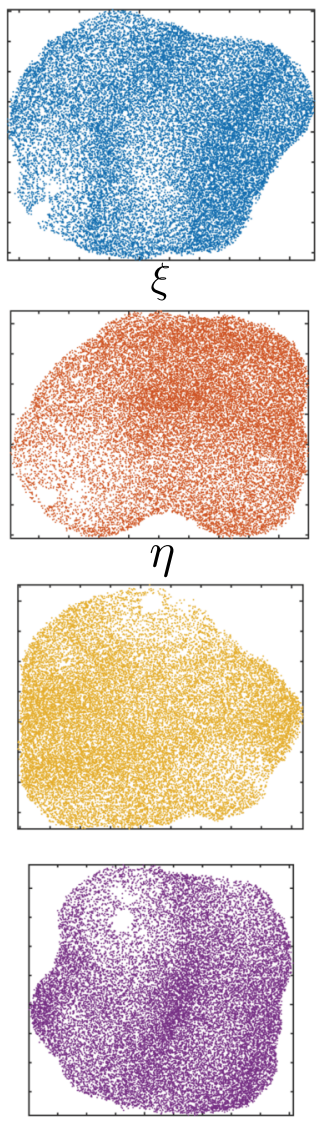

FIG. 4. Illustration of the partitioning of a training set of alanine dipeptide (a) into training sub-sets, which are subsequently embedded in low-dimensions using NLDR. Starting from a training set of molecular configurations (b), which can be represented in three dimensional space using the dihedral angles ( $\Phi$ and $\Psi)$ as a torus (c), we systematically partition the configurations into four slightly overlapping patches ((d), (e)). Then, by applying NLDR, we embed the conformations in each patch, devoid of topological obstructions, in low-dimensions (f).

$\psi_{\alpha}(\boldsymbol{r})>0$, we know that $C_{\alpha}$ is an active $\mathrm{CV}$ and if $\psi_{\alpha}(\boldsymbol{r})=1$, then it is the only active CV.

The ideas presented above provide a data-driven algorithmic framework to automatically construct an atlas of CVs for systems with general intrinsic manifold topology, without prior knowledge about the system other than a training set of conformations representative of molecular flexibility. We apply next this capability to free energy calculations using enhanced sampling along the atlas of CVs.

\section{B. Enhanced sampling with an atlas of collective variables}

The atlas of collective variables can be combined with a variety of enhanced sampling methods in various ways. For instance, different biased simulations could run independently in each of the partitions as in a stratification approach, and the trajectories could be combined to compute free energies adapting Weighted Histogram Analysis Method (WHAM) to the multi-CV framework recalling Eq. (5). In contrast, here, we develop a method in which a single biased MD simulation seamlessly transits across the different CVs. We consider a specific enhanced sampling method called adaptive biasing force $(\mathrm{ABF})$ method, ${ }^{40}$ although others such as metadynamics could be adapted as well.

During a biased MD trajectory, we identify for any configuration $\boldsymbol{r}$ the active charts, i.e., those whose partition of unity function $\psi_{\alpha}$ is greater than a small threshold. In general, because of overlap, each configuration has several active charts. Since bias forces from different active charts are different in general, cf., Eq. (9), we select a master chart, say $C_{1}$, to dictate the bias. This chart is selected at each time step following the criterion $\max _{\alpha} \psi_{\alpha}(\boldsymbol{r})$. All other active charts are tagged as slave. The biasing force is then computed as

$$
\boldsymbol{F}_{1}(\boldsymbol{r})=\mathbf{D} C_{1}^{T}(\boldsymbol{r}) \boldsymbol{f}_{1}(\boldsymbol{\xi}),
$$

where $f_{1}(\xi)$ is the biasing thermodynamic force conjugate to $\xi$ in the master chart and given by the ABF method. Next, we let all other active charts know about the exerted biasing force, and project this force along the corresponding CVs. Denoting $C_{2}$ a slave chart, we have

$$
\boldsymbol{f}_{2}(\boldsymbol{\eta})=\mathbf{D} \varphi^{-T}(\boldsymbol{\eta}) \boldsymbol{f}_{1}(\boldsymbol{\xi})
$$




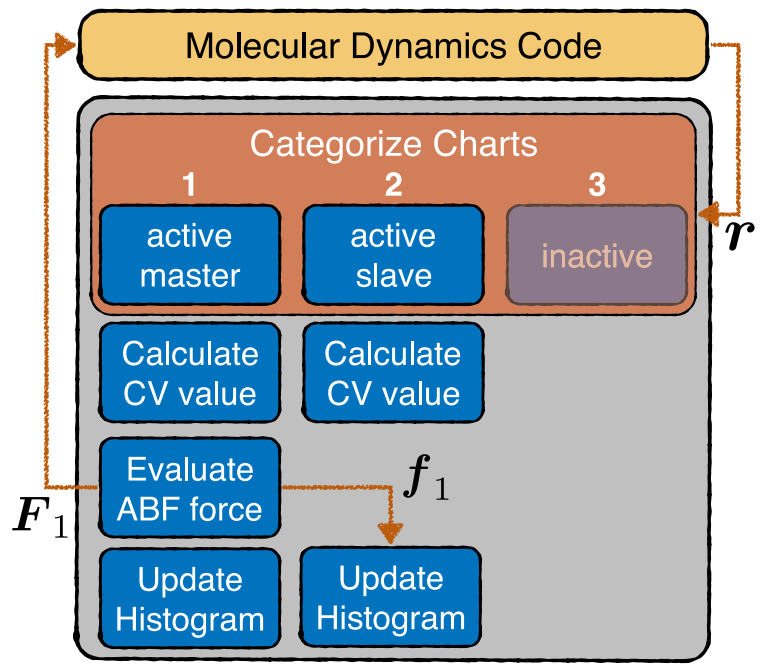

FIG. 5. Flowchart of an enhanced sampling simulation along an atlas of collective variables.

The biasing force $f_{1}(\xi)$ and its representation in slave charts $\boldsymbol{f}_{2}(\boldsymbol{\eta})$ are binned to generate a histogram of the mean thermodynamic force. Upon convergence of the algorithm, the free energy is reconstructed in each chart from the histograms as the potential of mean force. A visual summary of the procedure is shown in Figure 5. Thus, in this method there is a single trajectory but one independent free energy surface per chart. Yet, as shown in Section II, this multiple chart description is not an obstacle to computing thermodynamical observables.

\section{RESULTS}

We demonstrate next the concept of atlas of SandCVs, by implementing it in a standard MD code to perform enhanced sampling simulations and free-energy calculations. MD simulations were performed in version 2.8 of NAMD ${ }^{50}$ with Langevin thermostat at $300 \mathrm{~K}$. The atlas of SandCVs and the $\mathrm{ABF}$ algorithm for enhanced sampling are implemented in $\mathrm{C}++$ and communicate with NAMD through a TCL interface.

\section{A. Alanine dipeptide}

We focus first on alanine dipeptide (N-acetyl- $\mathrm{N}^{\prime}$ -methyl-L-alanylamide) in vacuum. We generate a training set using multiple short high-temperature MD runs ${ }^{33}$ with the CHARM22 force field. ${ }^{51}$ The actual partitioning and low-dimensional embedding used to build an atlas of CVs, as described in Sec. III, is shown in Figure 4. In Figure 6(a), we show the free energy surfaces (FES) along each of the four CVs systematically identified by the algorithm. The enhanced sampling MD trajectory seamlessly transitioned between charts and resulted in converged FES. The length of the trajectory required for convergence was comparable to that needed with a conventional ABF simulation based on backbone dihedrals. A direct comparison of the FES obtained with the atlas of CVs and with dihedral angles [Figure 6(d)] is not strictly meaningful because they differ by Fixmanlike terms. However, we can compare later thermodynamical observables obtained from these two different FES.

Our approach is obviously more expensive than a conventional ABF simulation based on backbone dihedral angles. On one hand, and leaving aside the multi-patch aspect of the method, evaluating data-based CVs, like SandCV, is more costly than evaluating an explicit CV. In SandCV, the main computation costs comes from the high-dimensional closest-point projection of a configuration onto the intrinsic manifold (and the derivatives of this operation). However, we previously showed that even this cost becomes insignificant in simulations involving larger systems, e.g., in explicit water. ${ }^{33}$ On the other hand, there is a computational overhead linked to the multi-patch nature of the method, as compared to a conventional single-chart $\mathrm{CV}$. We found that this specific overhead due to the atlas description is negligible. This can

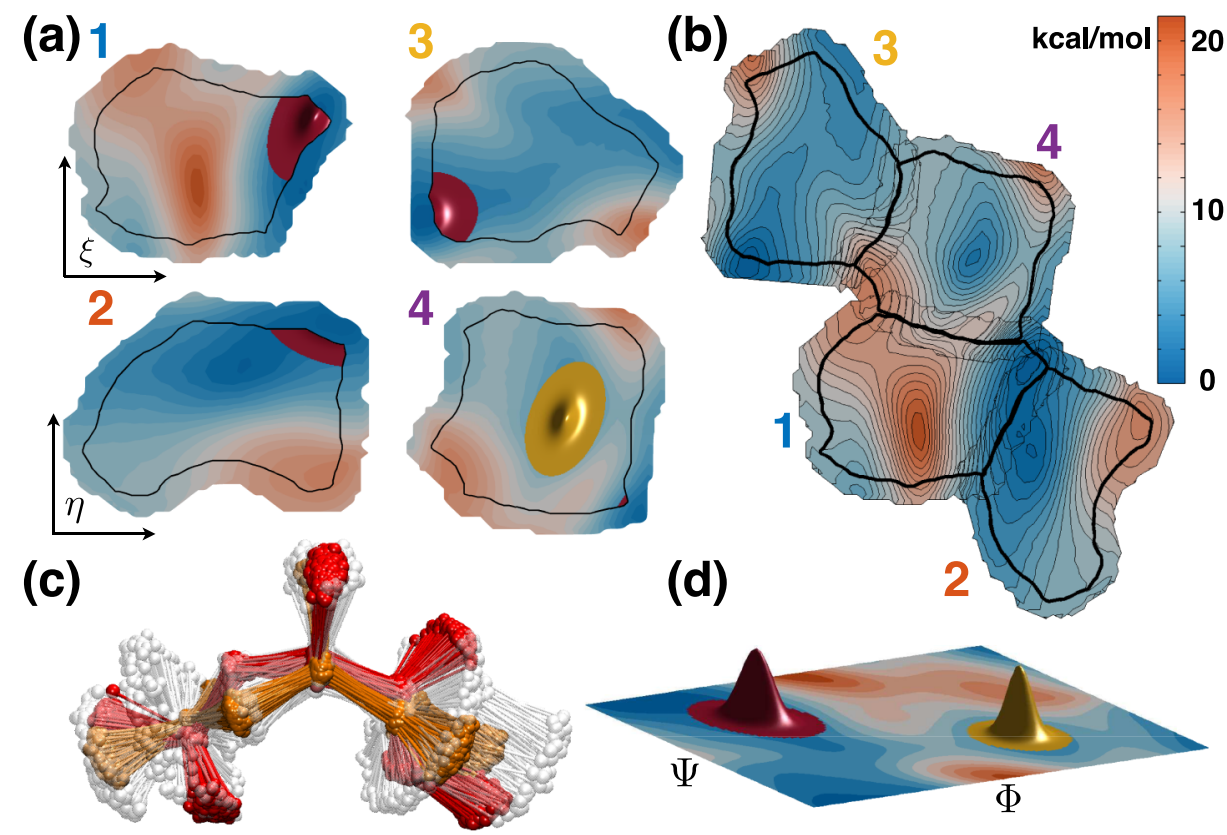

FIG. 6. Analysis with an atlas of CVs of alanine dipeptide. (a) Free energy surfaces computed for each of the four CVs resulting from the systematic partitioning in Figure 4, and obtained by a single trajectory seamlessly transiting between CVs. (b) Coherent juxtaposition of these free energy profiles, possible here because of the special flat geometry of conformational space of this molecule and the isometric character of Isomap. (c) Sampling of two states, $C_{7 e q}$ and $C_{7 a x}$, of the molecule. (d) Projection of these states in dihedral space, where we also computed the free energy surface using $\mathrm{ABF}$ along the two dihedral angles shown in Figure 4(a). 
be understood from the fact that the main extra computation is in the evaluation of Eq. (10), a low-dimensional matrix multiplication, and of the partition of unity functions, Eq. (A1), which involves calculating Euclidean distances to a few landmark configurations. Thus, the computational overhead of an enhanced simulation along an atlas of data-based CVs will not be significant for a large enough system.

Next, we discuss the role of the Fixman correction [Eq. (5)] in this example. Since the statistical error in the evaluation of the free energy is in the order of $k T$, Eq. (5) shows that the Fixman contribution is significant if $|\mathbf{D} \varphi|>e$, which for $d=2$ would require, for instance, stretching adjacent CV spaces by a factor of 1.6 along each coordinate. Interestingly, because of the nature of Isomap and the fact that the underlying intrinsic manifold is essentially flat (a flat torus), ${ }^{39}$ the four embeddings for this molecule are nearly isometric. This means that distances between low-dimensional points in Figure 4(f) are similar to distances of the corresponding conformations in high-dimensions [Figure 4(e)] and therefore close to the low-dimensional distances in an adjacent chart. As a result, $|\mathbf{D} \varphi| \approx 1$ and in this example the Fixman correction is negligible. Thus, we can graphically "glue" the different patches to visualize globally coherent FES, as illustrated in Figure 6(b). This observation favors building the atlas of CVs using the Isomap method for the NLDR, as compared to methods preserving other notions of high-dimensional distance.

We note however that such joint representation is not possible in general nor is it necessary to compute thermodynamic observables. To show this, we consider two states A and B, computationally described by two ensembles obtained by restrained MD simulations around conformations $C_{7 e q}$ and $C_{7 a x}$ of the molecule [Figure 6(c)]. For reference, these two states are graphically represented in dihedral space, where we also compute the FES [Figure 6(d)]. When represented in the atlas of CVs [Figure 6(a)], we note that $C_{7 e q}$ spreads over each of the four charts. Using Eq. (6) sampled at $1 \times 10^{6}$ configurations for each state, we find using the atlas of $\mathrm{CVs}$ that the probability of $C_{7 e q}$ relative to $C_{7 a x}$ is $P_{\text {atlas }}=56.406$. To sample integrals spanning multiple charts, we counted each sample only at its master chart. The excellent agreement with the same quantity computed using a separate free-energy surface defined over dihedral space, $P_{\text {dihed }}=56.415$, illustrates the accuracy of framework proposed here across multiple $\mathrm{CVs}$, and its potential to study more complex systems with nontrivial topology for which suitable CVs are not readily available.

\section{B. Six-membered rings}

We apply the proposed methodology to a different benchmark molecule, $\beta$-D-glucopyranose, ${ }^{4,5}$ known to have the topology of the sphere. ${ }^{52}$ For this six-membered ring, the Cremer-Pople puckering coordinates ${ }^{53}$ reduce to spherical coordinates $(Q, \theta, \phi)$, and adequately describe its molecular flexibility with small fluctuations about the "radius" $Q$. See Appendix B for details. To obtain a good training set, we performed a 10 ns metadynamics simulation with $\phi$ and $\theta$ as collective variables with Gaussian height of 0.1 and sigma of

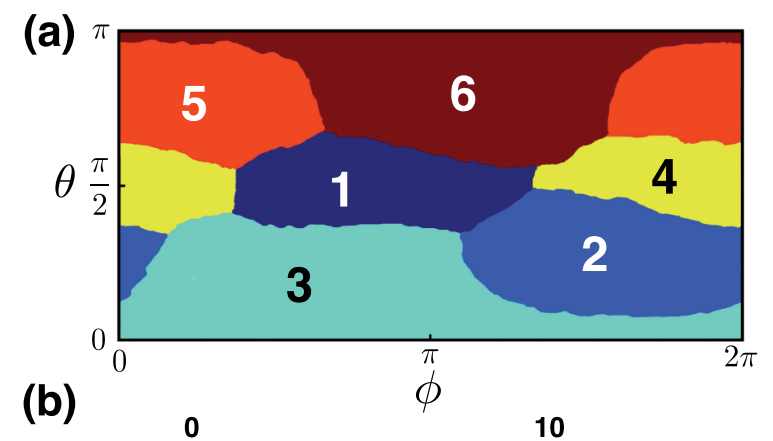

(b)

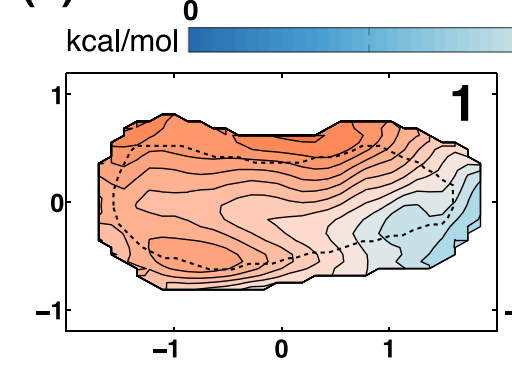

10
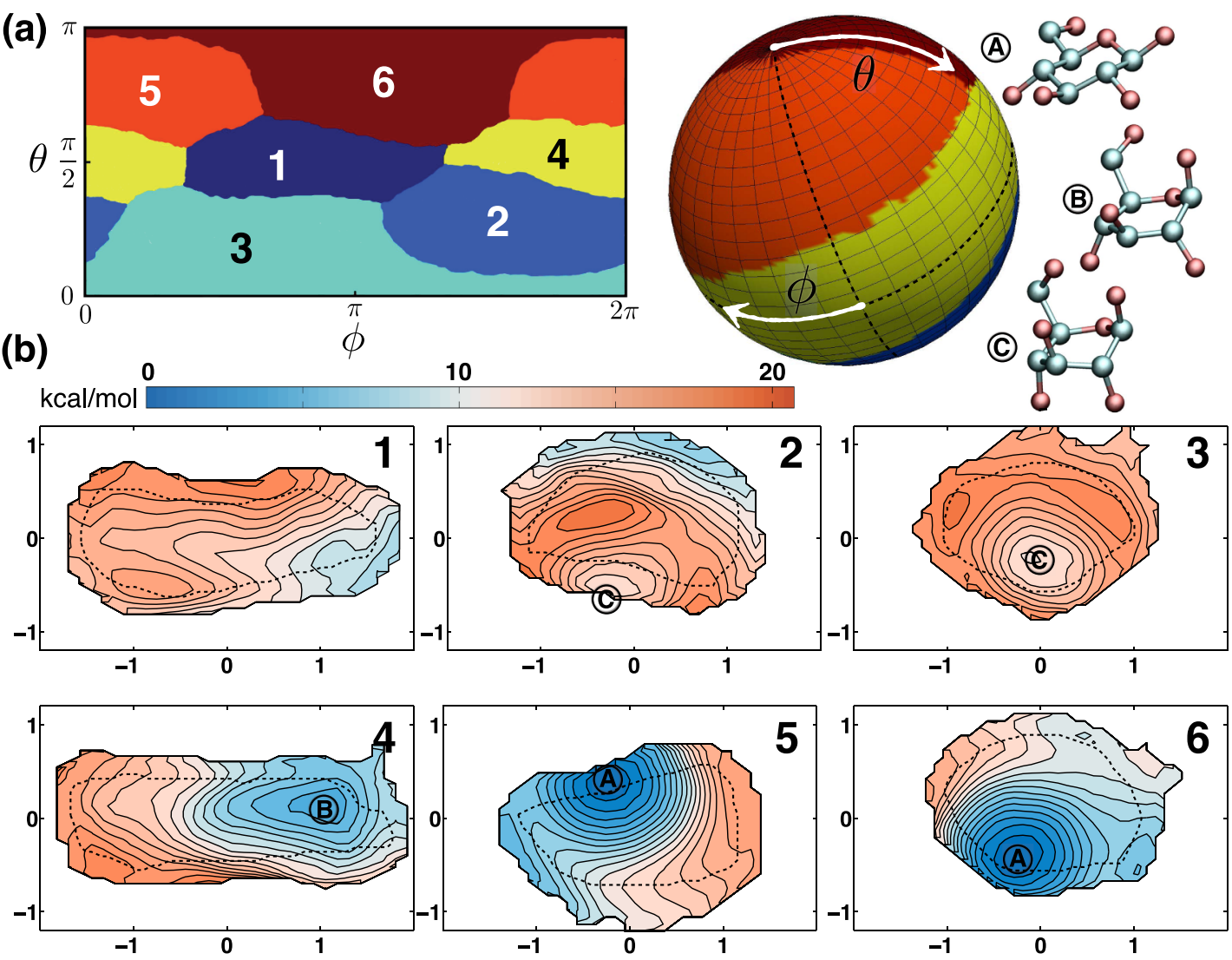

FIG. 7. Atlas of collective variables for $\beta$-D-glucopyranose, a six-membered ring carbohydrate. The systematic partitioning algorithm for this molecule results in six patches to overcome topological obstructions and alleviate geometric distortion (a), leading to six free-energy profiles (b). 
0.1 , starting from chair conformation $\left({ }^{4} C_{1}\right)$, and using Glycam force field ${ }^{54}$ at $300 \mathrm{~K}$ using Langevin thermostat. We set time step to $0.2 \mathrm{fs}$ and we sampled at each 500 steps (100 fs), obtaining $10^{5}$ configurations for training set.

The systematic partitioning of this training set led here to six different charts, as shown in Figure $7($ a) in $(\theta, \phi)$ and $(Q, \theta, \phi)$ spaces. Note that two partitions would be enough to remove the topological obstruction. However, the algorithm further subdivided the training set until the quality criteria given in Appendix A was met. Unlike the previous example, now the underlying two-dimensional conformational manifold is not flat, but rather intrinsically curved. With only two partitions, the low-dimensional embeddings in the plane significantly distort the actual distances between conformations, which in turn introduces significant Fixman terms as in Eq. (5). Instead, applying enhanced sampling along this atlas of six CVs, we find that the transition mappings have very little effect on the free-energy. The resulting FES are shown in Figure 7(b), where the dashed lines delimit the overlap region. Three selected conformations are placed on the FES to illustrate the connections between CVs. Because of the intrinsic curvature of the underlying manifold, it is not possible for this molecule to stitch these six FES along six local CVs without gaps or overlaps. However, as illustrated in the previous example, the atlas of CVs provides a self-standing statistical mechanics description of the system, and it is not necessary to glue these six FES to compute thermodynamics observables.

\section{CONCLUSION}

We have shown that molecular conformations and free energy landscapes can be described by an atlas of collective variables, in the same way that geographical landscapes are described by atlas of charts. Furthermore, we have provided a practical algorithm based on SandCVs ${ }^{33}$ to systematically implement this concept. This method builds a data-driven atlas of $\mathrm{CVs}$ identified from machine learning, which can be used to enhance sampling in MD simulations. The proposed framework generalizes the way we model molecular systems, by providing a globally meaningful thermodynamic description from locally appropriate CVs. The proposed framework could be attractive in systems that require different resolution in different regions of conformational space. More generally, the atlas of CVs provides a flexible framework for molecular modeling, which may help to better adapt the description of conformational landscapes to local features.

We have shown that this method overcomes some of the main limitations of CVs obtained from machine learning, such as their inability to deal with conformational manifolds of general topology such as those containing periodicities, very common in molecular systems, or the unavoidable geometric distortion introduced when trying to embed globally a curved manifold into a planar CV space. Thus, this method may also expand the applicability of data-driven CVs to systems with arbitrarily complex conformational manifold. We note, however, that an atlas of CVs could be constructed without resorting to machine learning, provided that the conditions identified in Section II are satisfied.

The application of the proposed method to simple yet nontrivial model systems suggests that the method could be applied to more complex molecules for which globally valid collective variables are not available. Such further studies will provide a clearer picture of the potential of the proposed methods. An important question not addressed here is whether a smooth atlas of CVs obtained from machine learning will provide a correct description of kinetic properties, i.e., whether it can be used to identify reaction coordinates. This can be tested using committor histograms. ${ }^{55}$ Interestingly, even for a simple reaction such as the isomerization of alanine dipeptide, the two dihedral angles $(\Phi, \Psi)$ cannot describe correctly the transition and at least three are required. ${ }^{56,57}$ However, while the two-dimensional description given by our method bears similarities with the two-dihedral description, it takes into account all atomic positions, and thus implicitly all dihedral angles in the molecule. Whether this non-locality inherent to SandCV is useful to correctly capture kinetics will be assessed in future studies.

\section{ACKNOWLEDGMENTS}

We acknowledge the support of the European Research Council under the European Community 7th Framework Programme (No. FP7/2007-2013)/ERC Grant Agreement No. 240487. We also thank Alejandro Torres for helpful discussions.

\section{APPENDIX A: ALGORITHM TO GEOMETRICALLY PARTITION THE CONFORMATIONAL MANIFOLD}

The atlas of collective variables approach relies on a systematic partitioning of the conformational manifold into pieces or patches that are tractable with dimensionality reduction techniques (open sets).

Consider a smooth $d$-manifold $\mathcal{M}$ embedded in $\mathbb{R}^{D}$ and sampled by a set of points $R=\left\{\boldsymbol{r}_{1}, \boldsymbol{r}_{2}, \ldots, \boldsymbol{r}_{N}\right\} \subset \mathbb{R}^{D}$, the conformations in the training set. The goal is to represent numerically $\mathcal{M}$ from the data in $R$ through a collection of overlapping parametrizations and make computations on it. ${ }^{49}$ Below are the steps involved in this algorithm:

1. Partition the set of points $R$ into $L$ groups on the basis of proximity using METIS domain decomposition with a $k$-nearest neighbor graph. ${ }^{48}$ METIS tries to partition a graph in equal size subdomains with minimal shared boundary lengths. These $L$ groups of points can be represented with index sets $\mathcal{I}_{\alpha}, \alpha=1, \ldots, L$ satisfying $\cup_{\alpha=1}^{L} \mathcal{I}_{\alpha}=\{1,2, \ldots, N\}$ and $\mathcal{I}_{\alpha} \cap \mathcal{I}_{\kappa}=\emptyset$ when $\alpha \neq \kappa$.

2. For each partition we create an enlarged index set $\mathcal{J}_{\alpha}$ by combining the indices in $\mathcal{I}_{\alpha}$ and the indices from their nearest neighbors defined by a cutoff distance $d_{\text {cut }}$,

$$
\mathcal{J}_{\alpha}=\left\{a \in\{1, \ldots, N\} \text { such that }\left|\boldsymbol{r}_{a}-\boldsymbol{r}_{b}\right|<d_{\text {cut }} \text { for some } b \in \mathcal{I}_{\alpha}\right\}
$$


Here and elsewhere, $|\cdot|$ denotes the Euclidean norm. The enlarged index set obeys $\cup_{\alpha=1}^{L} \mathcal{J}_{\alpha}=\{1,2, \ldots, N\}$, but now $\mathcal{J}_{\alpha} \cap \mathcal{J}_{\kappa} \neq \emptyset$. The intersection of these index sets defines the overlapping between different patches.

3. A dimensionality reduction technique is applied to each one of the enlarged sets $\left\{\boldsymbol{r}_{a}\right\}_{a \in \mathcal{J}_{\alpha}} \subset \mathbb{R}^{D}$ to find their low-dimensional embedding $\left\{\boldsymbol{\xi}_{a}\right\}_{a \in \mathcal{J}_{\alpha}} \subset \mathbb{R}^{d}$. In our calculations we use Isomap, a NLDR method designed to find a nearly isometric low-dimensional representation by approximately preserving the geodesic distance on the manifold.

4. The quality of the resulting embedding is measured through a reconstruction error computed as

$$
e_{a}=\frac{\left|\boldsymbol{r}_{a}-\sum_{b \in \mathcal{K}_{a}} w_{a b} \boldsymbol{r}_{b}\right|}{\left|\boldsymbol{r}_{a}\right|} \quad \forall a=1, \ldots, N,
$$

where $\mathcal{K}_{a}$ is the index set with the first $k$-nearest neighbors of the $a$ th configuration in the low-dimensional space, and $w_{a b}$ are the weights that best linearly reconstruct $\boldsymbol{\xi}_{a}$ from its $k$-nearest neighbors, ${ }^{27}$ obtained by

$$
\min _{w_{a b}}\left|\xi_{a}-\sum_{b \in \mathcal{K}_{a}} w_{a b} \boldsymbol{\xi}_{b}\right| \quad \text { subject to } \sum_{b \in \mathcal{K}_{a}} w_{a b}=1 .
$$

5. We monitor in each patch the reconstruction error. We require that $\max \left\{e_{a}\right\}_{a \in \mathcal{J}_{\alpha}}<$ Tol $_{e}$ for a patch to be acceptable, where $\mathrm{Tol}_{e}$ is a numerical tolerance typically below 0.1. If the reconstruction error exceeds the tolerance, the patch is recursively subdivided until the reconstruction requirement is met. In Figure 8, we show the reconstruction error for different number of patches of a training set of alanine dipeptide.

6. After the partitioning process is finished and the lowdimensional embedding for each partition is available, we

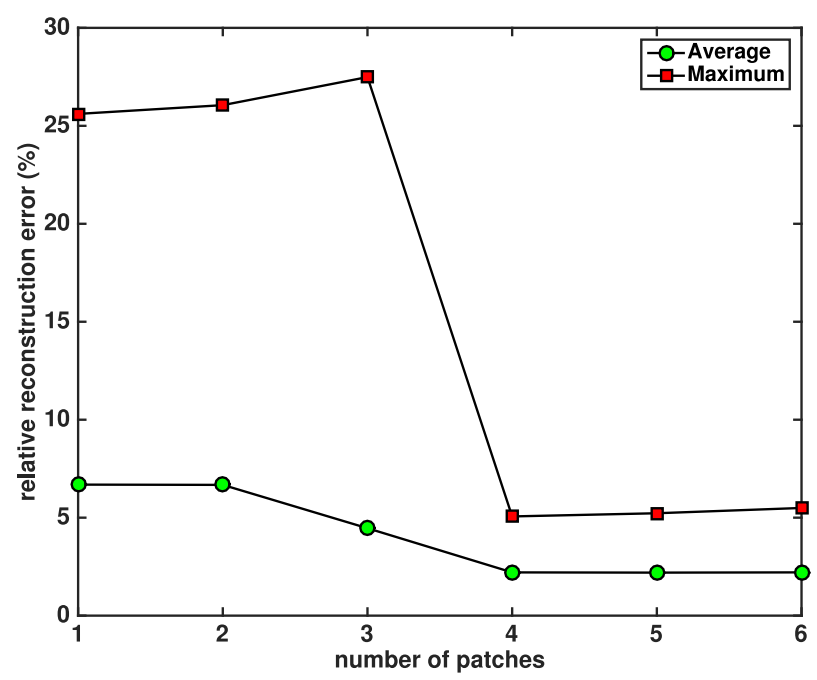

FIG. 8. Average (green circles) and maximum (red squares) relative reconstruction error in the partitioning of alanine dipeptide as a function of the number of patches in the partition. It is clear from this plot that the maximum norm best discriminates the number of required patches given by the algorithm, four in this case.

build a smooth parametrization for each partition using smooth basis functions $p_{a}(\xi)$ associated to nodes from a uniform grid of landmarks,

$$
\begin{aligned}
\mathcal{M}_{\alpha}: \Omega_{\alpha} \subset \mathbb{R}^{d} & \longrightarrow \mathbb{R}^{D} \\
\boldsymbol{\xi} & \longmapsto \sum_{a \in \mathcal{J}_{\alpha}} p_{a}(\boldsymbol{\xi}) \boldsymbol{y}_{a} .
\end{aligned}
$$

The high-dimensional control points $\boldsymbol{y}_{a} \in \mathbb{R}^{D}$ are chosen such that the reconstruction error is minimized in a leastsquares sense. Full details are presented in Ref. 33. Thus,

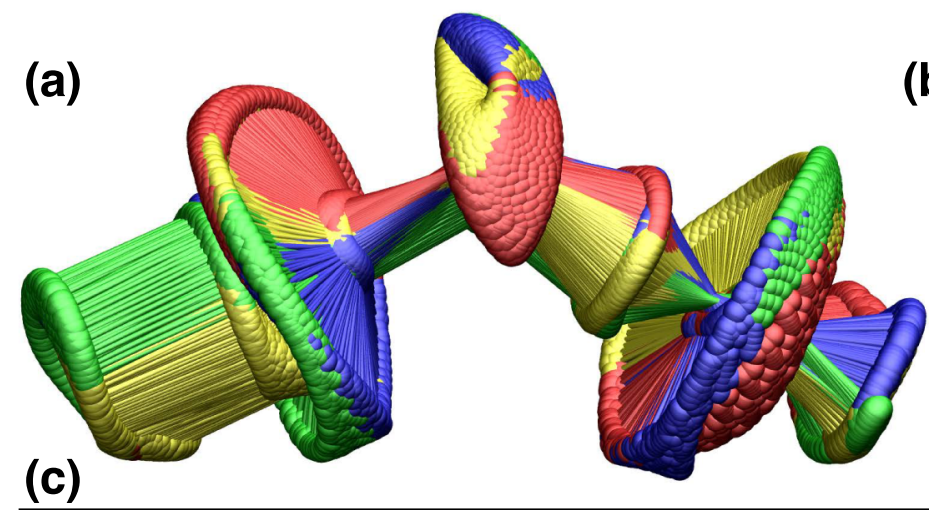

(b)

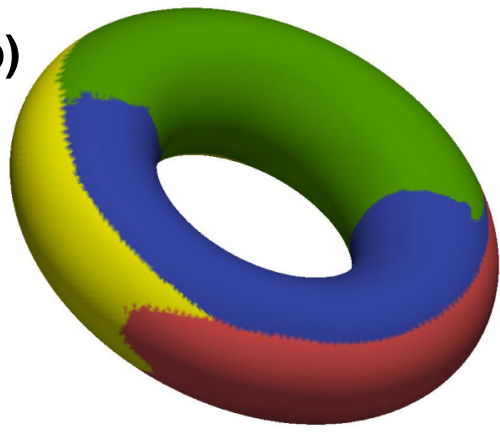

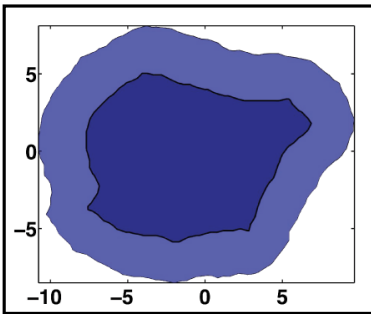
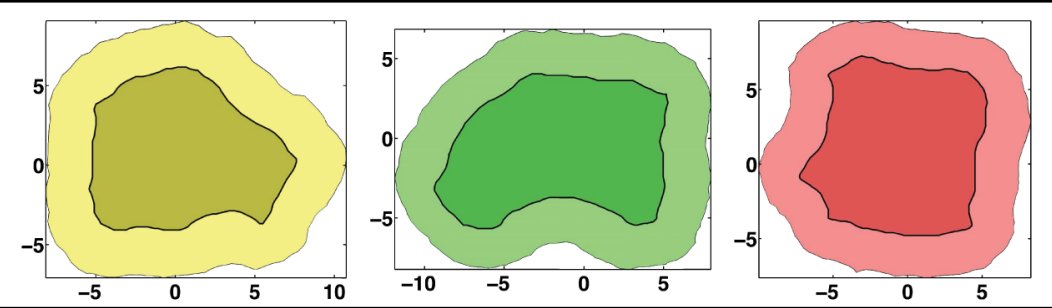

FIG. 9. Partitioning a training set of alanine dipeptide. The partitioning procedure recursively proceeds until all the patches in the partition are tractable with nonlinear dimensionality reduction (NLDR) methods, which leads to four patches in this example. Although the patches have overlap, using a partition of unity we can unambiguously assign a single master patch to any conformation (a). These patches are also depicted over a torus of dihedral angles to highlight the topology of the intrinsic manifold (b). The low-dimensional embedding of each of the four patches of the partition, calculated separately with NLDR methods, is shown in (c). The darker colors mark the low-dimensional representation of conformations into their master patch. 


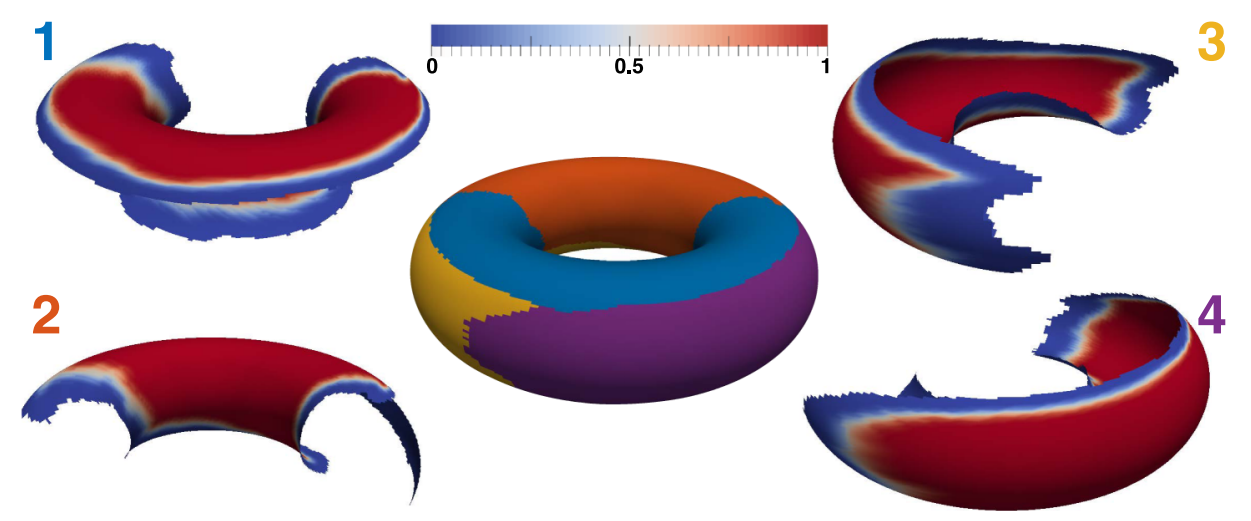

FIG. 10. Partition of unity for alanine dipeptide. Partition of unity functions are represented on the torus defined by dihedral angles. It is worth emphasizing that these functions are evaluated in high-dimensional configuration space and the torus representation is for visualization purposes only.

we end up with a collection of partially overlapping parametrizations of the intrinsic manifold, Figure 9. We consider here the local maximum-entropy (LME) basis functions. ${ }^{58-60}$

A partition of unity subordinates to the geometric partition of the conformational manifold is a set of non-negative functions $\psi_{\alpha}(\boldsymbol{r})$ that add up to 1 everywhere, and whose support is contained in the respective patch. Given a set of non-negative reals $\left\{\beta_{a}\right\}_{a=1,2, \ldots, N}$, we consider the Shepard partition of unity with Gaussian weight associated to patch $\alpha$ as

$$
\psi_{\alpha}(\boldsymbol{r})=\frac{\sum_{a \in I_{\alpha}} \exp \left(-\beta_{a}\left|\boldsymbol{r}-\boldsymbol{r}_{a}\right|^{2}\right)}{\sum_{b=1}^{N} \exp \left(-\beta_{b}\left|\boldsymbol{r}-\boldsymbol{r}_{b}\right|^{2}\right)} .
$$

These non-negative functions form a partition of unity in $\mathbb{R}^{D}$. For very large $\beta_{a}$, these functions tend to the characteristic functions of the Voronoi cells in high-dimension associated to the group of points given by $\mathcal{I}_{\alpha}$. They can thus be viewed as a smooth regularization of these characteristic functions. ${ }^{49}$ The four partition of unity functions in the torus representation of alanine dipeptide is illustrated in Figure 10. These partition of unity functions also divide high-dimensional configuration space into non-overlapping regions, i.e., they provide a definition of the master patch associated to $\boldsymbol{r}$ maximizing $\psi_{\alpha}(\boldsymbol{r})$ with respect to $\alpha$. A chart or $\mathrm{CV}$ is associated to each patch by the closest-point projection as discussed in the main text.

\section{APPENDIX B: ON PUCKERING COORDINATE OF SIX-MEMBERED RINGS}

The conformation of any $N$-membered ring can be described univocally by Cremer-Pople puckering coordinates. ${ }^{53}$ For six-membered rings $(N=6)$, like $\beta$-Dglucopyranose, there are three puckering degrees of freedom, which admit a representation in spherical coordinates as

$$
\begin{aligned}
& Q=\sqrt{\sum_{j=1}^{6} z_{j},} \\
& \theta=\cos ^{-1}\left[\frac{\sqrt{\frac{1}{6} \sum_{j=1}^{6} z_{j}(-1)^{j-1}}}{\sqrt{\sum_{j=1}^{6} z_{j}}}\right], \\
& \phi=\tan ^{-1}\left\{\frac{\sum_{j=1}^{6} z_{j} \sin \left[\frac{2 \pi}{3}(j-1)\right]}{\sum_{j=1}^{6} z_{j} \cos \left[\frac{2 \pi}{3}(j-1)\right]},\right.
\end{aligned}
$$

where $z_{j}$ is defined from the position of atoms, $\boldsymbol{x}_{i}$,

$$
\begin{aligned}
z_{j} & =\mathbf{R}_{j} \cdot \hat{\boldsymbol{n}}, \\
\mathbf{R}_{j} & =\sum_{i=1}^{6} \boldsymbol{x}_{i}\left(\delta_{i j}-1 / N\right), \quad \hat{\boldsymbol{n}}=\frac{\mathbf{R}^{\prime} \times \mathbf{R}^{\prime \prime}}{\left|\mathbf{R}^{\prime} \times \mathbf{R}^{\prime \prime}\right|}, \\
\mathbf{R}^{\prime} & =\sum_{j=1}^{6} \mathbf{R}_{j} \sin (\pi(j-1) / 3), \\
\mathbf{R}^{\prime \prime} & =\sum_{j=1}^{6} \mathbf{R}_{j} \cos (\pi(j-1) / 3) .
\end{aligned}
$$

In Cartesian coordinates, these puckering coordinates can be expressed as

$$
\begin{aligned}
& q_{x}=Q \sin \theta \sin \phi, \\
& q_{y}=Q \sin \theta \cos \phi, \\
& q_{z}=Q \cos \theta .
\end{aligned}
$$

The relationship between these two representations of puckering coordinates is shown in Figure 11(a). Since $\beta$-Dglucopyranose evolves close to a spherical intrinsic manifold, only two independent parameters are needed to define a CV. In enhanced sampling simulations and free energy calculations, two different CVs are commonly used, based on either polar coordinates $C_{1}=(\theta, \phi),{ }^{61}$ Figure 11(b), or Cartesian coordinates $C_{2}=\left(q_{x}, q_{y}\right),{ }^{52}$ Figure 11(c). The latter can be only defined in one hemisphere to avoid data collapse as a result of a topological obstruction. ${ }^{39}$

Comparing the free energy along these CVs is not trivial. If we assume that there is no variation in the $Q$-direction and the system stays exactly on the intrinsic manifold (the sphere), we can define a bijective mapping between CVs from Eq. (B1) and evaluate the Fixman correction between the corresponding free energy surfaces. In Figure 11(d), we show that this correction, along $\theta$ and for any value of $\phi$, is significant particularly at the pole and equator. In reality, although the variation along $Q$ is small compared to $\theta$ and $\phi,{ }^{52}$ it is not negligible. Because the level sets of $C_{1}$ and $C_{2}$ are fundamentally different, the portion of configuration space that is integrated is also different, see Figure 11(e), and thus quantitatively comparing the free energies is meaningless (although observables computed from them are well defined). The Cartesian projection of a trajectory when $Q$ is allowed to fluctuate is illustrated in Figure 11(f). 
(a)

(d)
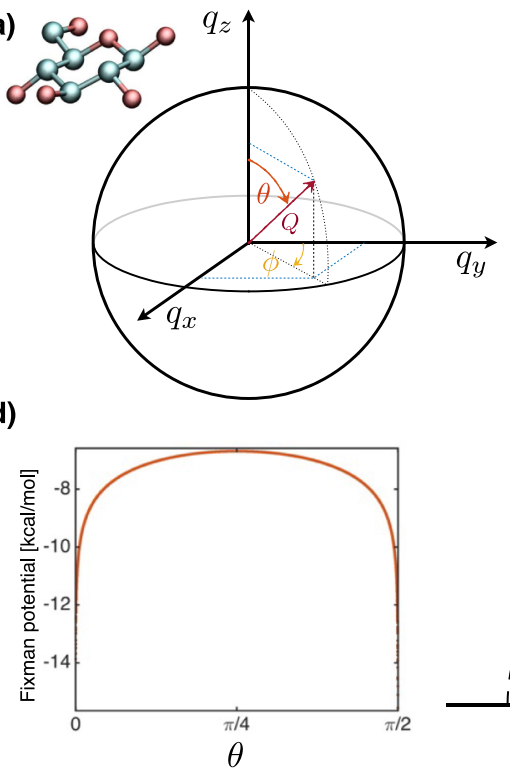

(b)

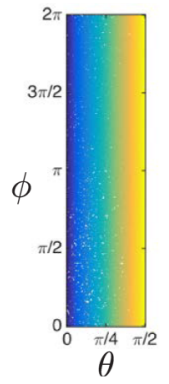

(e)

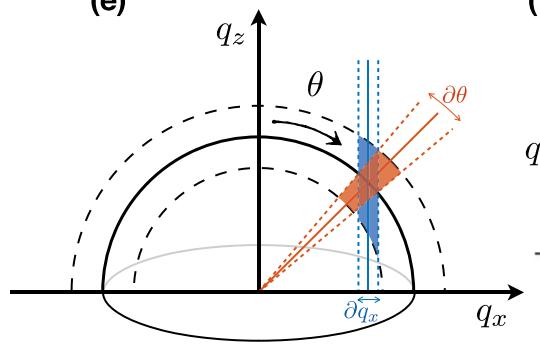

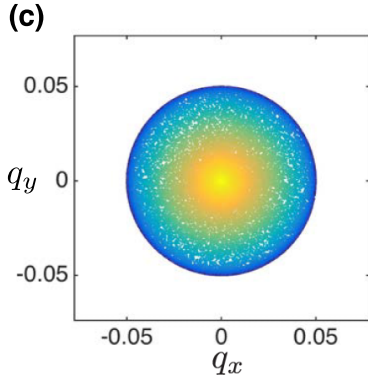

(f)

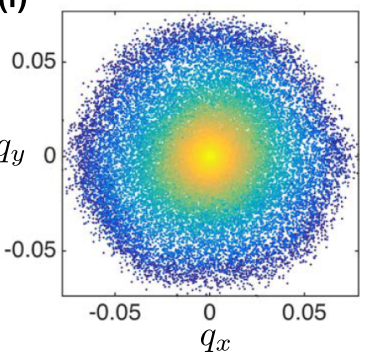

FIG. 11. Cremer-Pople puckering coordinates for six-membered rings is given either by $\left(q_{x}, q_{y}, q_{z}\right)$ in Cartesian coordinates or by $(Q, \theta, \phi)$ in spherical coordinates (a). Two common collective variables in enhanced sampling MD and free energy calculations are $C_{1}=(\theta, \phi)(\mathrm{b})$ and $C_{2}=\left(q_{x}, q_{y}\right)(\mathrm{c})$. Fixman correction associated with the change of variables between these CVs while staying on the intrinsic manifold, i.e., $Q$ fixed (d). Different portions of configuration space visited by fixing each of these two CVs (e) when there is a variation along $Q$ direction (f).

${ }^{1}$ M. Ceriotti, G. A. Tribello, and M. Parrinello, J. Chem. Theory Comput. 9, 1521-1532 (2013).

${ }^{2}$ G. A. Tribello, M. Ceriotti, and M. Parrinello, Proc. Natl. Acad. Sci. U. S. A. 107, 17509-17514 (2010).

${ }^{3}$ B. M. Dickson, D. E. Makarov, and G. Henkelman, J. Chem. Phys. 131, 074108 (2009).

${ }^{4}$ V. Spiwok, B. Králová, and I. Tvaroska, Carbohydr. Res. 345, 530-537 (2010).

${ }^{5}$ D. Branduardi and J. D. Faraldo-Gómez, J. Chem. Theory Comput. 9, 4140-4154 (2013).

${ }^{6}$ A. L. Ferguson, A. Z. Panagiotopoulos, P. G. Debenedetti, and I. G. Kevrekidis, J. Chem. Phys. 134, 135103 (2011).

${ }^{7}$ M. Ceriotti, G. A. Tribello, and M. Parrinello, Proc. Natl. Acad. Sci. U. S. A. 108, 13023-13028 (2011).

${ }^{8}$ A. Amadei, A. B. Linssen, and H. J. C. Berendsen, Proteins 17, 412-425 (1993).

${ }^{9}$ P. Das, M. Moll, H. Stamati, L. E. Kavraki, and C. Clementi, Proc. Natl. Acad. Sci. U. S. A. 103, 9885-9890 (2006).

${ }^{10}$ W. Zheng, B. Qi, M. A. Rohrdanz, A. Caflisch, A. R. Dinner, and C. Clementi, J. Phys. Chem. B 115, 13065-13074 (2011).

${ }^{11}$ Springer Series in Chemical Physics, edited by C. Chipot and A. Pohorille (Springer, Berlin, Heidelberg, 2007), Vol. 86.

${ }^{12}$ M. Salvalaglio, P. Tiwary, and M. Parrinello, J. Chem. Theory Comput. 10, 1420-1425 (2014).

${ }^{13}$ J. Lu and E. Vanden-Eijnden, J. Chem. Phys. 141, 044109 (2014).

${ }^{14}$ E. Rosta, H. L. Woodcock, B. R. Brooks, and G. Hummer, J. Comput. Chem. 30, 1634-1641 (2009).

${ }^{15}$ L. Sutto, S. Marsili, and F. L. Gervasio, Wiley Interdiscip. Rev.: Comput. Mol. Sci. 2, 771-779 (2012)

${ }^{16} \mathrm{~J}$. A. Lee and M. Verleysen, Nonlinear Dimensionality Reduction, Information Science and Statistics (Springer, New York, NY, 2007).

${ }^{17}$ B. L. de Groot, X. Daura, A. E. Mark, and H. Grubmüller, J. Mol. Biol. 309, 299-313 (2001).

${ }^{18}$ O. F. Lange and H. Grubmüller, J. Phys. Chem. B 110, 22842-22852 (2006).

${ }^{19}$ G. G. Maisuradze, A. Liwo, and H. A. Scheraga, J. Mol. Biol. 385, 312-329 (2009).

${ }^{20}$ C. C. David and D. J. Jacobs, Methods Mol. Biol. 1084, 193-226 (2014).

${ }^{21}$ V. Spiwok, P. Lipovová, and B. Králová, J. Phys. Chem. B 111, 3073-3076 (2007).

${ }^{22}$ S. Michielssens, T. S. van Erp, C. Kutzner, A. Ceulemans, and B. L. de Groot, J. Phys. Chem. B 116, 8350-8354 (2012).

${ }^{23}$ A. García, Phys. Rev. Lett. 68, 2696-2699 (1992).

${ }^{24}$ H. Noji, R. Yasuda, M. Yoshida, and K. Kinosita, Nature 386, 299-302 (1997).
${ }^{25}$ R. Hegger, A. Altis, P. Nguyen, and G. Stock, Phys. Rev. Lett. 98, 028102 (2007).

${ }^{26}$ J. B. Tenenbaum, V. de Silva, and J. C. Langford, Science 290, 2319-2323 (2000).

${ }^{27}$ S. T. Roweis and L. K. Saul, Science 290, 2323-2326 (2000).

${ }^{28}$ G. E. Hinton and R. R. Salakhutdinov, Science 313, 504-507 (2006).

${ }^{29}$ W. M. Brown, S. Martin, S. N. Pollock, E. A. Coutsias, and J.-P. Watson, J. Chem. Phys. 129, 064118 (2008).

${ }^{30}$ A. L. Ferguson, A. Z. Panagiotopoulos, P. G. Debenedetti, and I. G. Kevrekidis, Proc. Natl. Acad. Sci. U. S. A. 107, 13597-13602 (2010).

${ }^{31}$ H. Stamati, C. Clementi, and L. E. Kavraki, Proteins 78, 223-235 (2010).

${ }^{32}$ V. Spiwok and B. Králová, J. Chem. Phys. 135, 224504 (2011).

${ }^{33}$ B. Hashemian, D. Millán, and M. Arroyo, J. Chem. Phys. 139, 214101 (2013).

${ }^{34}$ R. R. Coifman and S. Lafon, Appl. Comput. Harmonic Anal. 21, 5-30 (2006).

${ }^{35}$ M. A. Rohrdanz, W. Zheng, M. Maggioni, and C. Clementi, J. Chem. Phys. 134, 124116 (2011)

${ }^{36}$ G. A. Tribello, M. Ceriotti, and M. Parrinello, Proc. Natl. Acad. Sci. U. S. A. 109, 5196-5201 (2012).

${ }^{37}$ Y. Xue, P. J. Ludovice, M. A. Grover, L. V. Nedialkova, C. J. Dsilva, and I. G. Kevrekidis, Comput. Chem. Eng. 51, 102-110 (2013).

${ }^{38}$ M. Duan, M. Li, L. Han, and S. Huo, Proteins 82, 2585-2596 (2014)

${ }^{39}$ B. Hashemian and M. Arroyo, J. Chem. Phys. 142, 044102 (2015).

${ }^{40}$ E. Darve, D. Rodríguez-Gómez, and A. Pohorille, J. Chem. Phys. 128, 144120 (2008)

${ }^{41}$ T. Lelièvre, M. Rousset, and G. Stoltz, Free Energy Computations (Imperial College Press, London, 2010).

${ }^{42}$ C. Hartmann, J. C. Latorre, and G. Ciccotti, Eur. Phys. J.: Spec. Top. 200, 73-89 (2011).

${ }^{43}$ A. Ben-Israel, Linear Algebra Its Appl. 167, 87-111 (1992).

${ }^{44}$ D. Frenkel, Eur. Phys. J. Plus 128, 10 (2013).

${ }^{45}$ M. Fixman, Proc. Natl. Acad. Sci. U. S. A. 71, 3050-3053 (1974).

${ }^{46}$ A. Laio and M. Parrinello, Proc. Natl. Acad. Sci. U. S. A. 99, 12562-12566 (2002).

${ }^{47}$ V. Spiwok, P. Oborský, J. Pazúriková, A. Křenek, and B. Králová, J. Chem. Phys. 142, 115101 (2015).

${ }^{48}$ G. Karypis and V. Kumar, SIAM J. Sci. Comput. 20, 359-392 (1998).

${ }^{49}$ D. Millán, A. Rosolen, and M. Arroyo, Int. J. Numer. Methods Eng. 93, 685-713 (2013).

${ }^{50}$ J. C. Phillips, R. Braun, W. Wang, J. Gumbart, E. Tajkhorshid, E. Villa, C. Chipot, R. D. Skeel, L. Kalé, and K. Schulten, J. Comput. Chem. 26, 1781-1802 (2005). 
${ }^{51}$ A. D. MacKerell, D. Bashford, R. L. Dunbrack, J. D. Evanseck, M. J. Field, S. Fischer, J. Gao, H. Guo, S. Ha, D. Joseph-McCarthy, L. Kuchnir, K. Kuczera, F. T. K. Lau, C. Mattos, S. Michnick, T. Ngo, D. T. Nguyen, B. Prodhom, W. E. Reiher, B. Roux, M. Schlenkrich, J. C. Smith, R. Stote, J. Straub, M. Watanabe, J. Wiórkiewicz-Kuczera, D. Yin, and M. Karplus, J. Phys. Chem. B 102, 3586-3616 (1998).

${ }^{52}$ X. Biarnés, A. Ardèvol, A. Planas, C. Rovira, A. Laio, and M. Parrinello, J. Am. Chem. Soc. 129, 10686-10693 (2007).

${ }^{53}$ D. Cremer and J. A. Pople, J. Am. Chem. Soc. 97, 1354-1358 (1975).

${ }^{54}$ K. N. Kirschner, A. B. Yongye, S. M. Tschampel, J. González-Outeiriño, C. R. Daniels, B. L. Foley, and R. J. Woods, J. Comput. Chem. 29, 622-655 (2008).
${ }^{55}$ W. Li and A. Ma, Mol. Simul. 40, 784-793 (2014).

${ }^{56}$ P. G. Bolhuis, C. Dellago, and D. Chandler, Proc. Natl. Acad. Sci. U. S. A. 97, 5877-5882 (2000)

${ }^{57}$ L. Maragliano, A. Fischer, E. Vanden-Eijnden, and G. Ciccotti, J. Chem. Phys. 125, 24106 (2006).

${ }^{58}$ M. Arroyo and M. Ortiz, Int. J. Numer. Methods Eng. 65, 2167-2202 (2006).

${ }^{59}$ A. Rosolen, D. Millán, and M. Arroyo, Int. J. Numer. Methods Eng. 82, 868-895 (2010).

${ }^{60}$ D. Millán, A. Rosolen, and M. Arroyo, Int. J. Numer. Methods Eng. 85, 723-751 (2011).

${ }^{61}$ M. Sega, E. Autieri, and F. Pederiva, J. Chem. Phys. 130, 225102 (2009). 